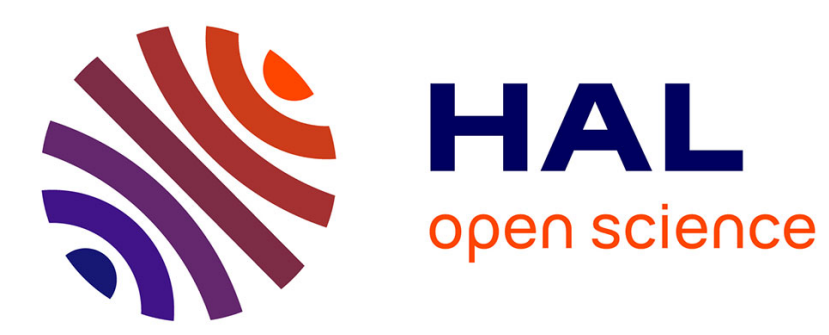

\title{
Mesure par interférométrie laser du mouvement d'une particule proche d'une paroi
}

Y. Assou, Denis Joyeux, A. Azouni, F. Feuillebois

\section{To cite this version:}

Y. Assou, Denis Joyeux, A. Azouni, F. Feuillebois. Mesure par interférométrie laser du mouvement d'une particule proche d'une paroi. Journal de Physique III, 1991, 1 (2), pp.315-330. 10.1051/jp3:1991125 . jpa-00248583

\section{HAL Id: jpa-00248583 https://hal.science/jpa-00248583}

Submitted on 1 Jan 1991

HAL is a multi-disciplinary open access archive for the deposit and dissemination of scientific research documents, whether they are published or not. The documents may come from teaching and research institutions in France or abroad, or from public or private research centers.
L'archive ouverte pluridisciplinaire HAL, est destinée au dépôt et à la diffusion de documents scientifiques de niveau recherche, publiés ou non, émanant des établissements d'enseignement et de recherche français ou étrangers, des laboratoires publics ou privés. 
Classification

Physics Abstracts

$07.60 \mathrm{~L}-47.80-81.40 \mathrm{P}$

\title{
Mesure par interférométrie laser du mouvement d'une particule proche d'une paroi .
}

\author{
Y. Assou ( ${ }^{1}$ ), D. Joyeux $\left({ }^{2}\right)$, A. Azouni $\left({ }^{1}\right)$ et F. Feuillebois ( $\left.{ }^{1}\right)$ \\ (1) Laboratoire d'Aérothermique du C.N.R.S., 4 ter Route des Gardes, F-92190 Meudon, \\ France \\ (2) Institut d'Optique (CNRS, UA 14), BP 147, F-91403 Orsay Cedex, France
}

(Reçu le 12 mars 1990, révisé le 6 septembre 1990, accepté le 29 octobre 1990)

\begin{abstract}
Résumé. - On utilise une technique expérimentale basée sur l'interférométrie laser pour déterminer le déplacement d'une sphère s'approchant d'une paroi plane dans un fluide visqueux : la technique consiste à insérer la sphère dans un système interférométrique, de façon que son déplacement change l'état d'interférence détecté. Une particularité importante du montage utilisé est que la forme, l'état de surface, le positionnement, et l'alignement de la sphère dans le montage optique sont relativement peu critiques. Les sphères utilisées sont des billes de roulements à billes, de 4 et $5 \times 10^{-3} \mathrm{~m}$ de diamètre. La précision sur le déplacement de la sphère est de l'ordre de $2 \times 10^{-8} \mathrm{~m}$. Le signal d'interférence est digitalisé et stocké dans un micro-ordinateur. Le traitement des données permet alors d'obtenir le coefficient de frottement $f_{z z}^{\mathrm{T}}$ de la sphère en mouvement tout près de la paroi. La variation de $f_{z z}^{\mathrm{T}}$ avec l'intervalle sans dimension $\varepsilon$ (rapport de l'intervalle entre la sphère et la paroi au rayon de la sphère) permet de distinguer trois régions : (i) Une région où $\varepsilon$ est petit mais au moins 10 fois plus grand que la rugosité non dimensionnelle (rapport de l'échelle de la rugosité au rayon de la sphère); le résultat expérimental est alors en très bon accord avec la formule $f_{z z}^{\mathrm{T}}=1 / \varepsilon$ démontrée en théorie de la lubrification, formule valable pour une sphère lisse. (ii) Lorsque $\varepsilon$ décroît pour devenir de l'ordre de grandeur de la rugosité non dimensionnelle, le coefficient de frottement est alors inférieur à $1 / \varepsilon$. La technique ouvre ainsi une nouvelle voie à l'étude des effets de rugosité en hydrodynamique. (iii) La technique permet de mesurer des intervalles de l'ordre de $10^{-8} \mathrm{~m}$ et pourrait ainsi être utilisée dans l'étude des forces à courte portée.
\end{abstract}

\footnotetext{
Abstract. - An experimental technique based on laser interferometry is used to obtain the displacement of a sphere towards a plane wall in a viscous fluid : it consists in inserting the sphere in an interferometric system, in such a way that the displacement of the sphere changes the state of interference. An important feature of the setup is that the shape, the roughness, and the positioning of the sphere in the optical path are relatively uncritical. The spheres used in the experiment are bearing balls, 4 and $5 \times 10^{-3} \mathrm{~m}$ in diameter. The accuracy on the sphere displacement is of the order of $2 \times 10^{-8} \mathrm{~m}$. The interference signal is digitalized and stored in a microcomputer. The data are then processed to yield the friction coefficient $f_{z z}^{\mathrm{T}}$ of the sphere in its motion very close to the wall. The variation of $f_{z z}^{\mathrm{T}}$ with the non-dimensional gap $\varepsilon$ (ratio of the gap to the sphere radius) show three regions : (i) One in which $\varepsilon$ is small but at least 10 times larger than the non-dimensional rugosity (ratio of the scale of rugosity to the sphere radius); there is then a very good agreement with the result valid for a smooth sphere $f_{z z}^{\mathrm{T}}=1 / \varepsilon$ from lubrication theory. (ii) When $\varepsilon$ decreases to the order of the non-dimensional rugosity, the friction coefficient
} 
is then smaller than $1 / \varepsilon$. The technique thus opens a way to study the effects of rugosity in hydrodynamics. (iii) The technique allows to measure gaps of the order of $10^{-8} \mathrm{~m}$ and could thus be used in future studies of short ranges forces.

\section{Introduction.}

1.1 Conditions EXPÉrimentales. - Pour décrire le mouvement de suspensions de particules solides dans un fluide visqueux, il faut précisèr les conditions aux limites sur les parois. Il est en particulier nécessaire de modéliser l'arrivée et le « choc » d'une particule sur une paroi plane. Ce problème a différentes applications en génie civil, dans différents processus industriels en génie chimique, ...

Nous considérons ici une particule solide et sphérique en chute libre dans un fluide visqueux au repos et se dirigeant vers une paroi solide plane et horizontale. Lorsque la particule s'approche de la paroi, l'écoulement autour de la particule est modifié. La force hydrodynamique èt donc la trajectoire de la particule s'en trouvent altérées.

Le nombre de Reynolds de l'écoulement par rapport à la particule est supposé petit. Ceci est le cas par exemple pour de petites particules (inférieures à $10^{-4} \mathrm{~m}$ ) en suspension dans de l'air ou de l'eau.

Cet écoulement à faible nombre de Reynolds peut être modélisé dans une expérience utilisant des particules plus grosses (de l'ordre de $10^{-3} \mathrm{~m}$ ) dans une huile très visqueuse. Il est alors possible d'observer plus en détail le mouvement de la sphère au voisinage immédiat de la paroi. Ceci a été fait par différents auteurs et notre travail utilise aussi cette modélisation.

1.2 EXPÉRIENCES ANTÉRIEURES. - Yuu et Fukui [7] et Adamczyk et al. [1] ont observé le mouvement d'une sphère solide lâchée au-dessus d'une paroi plane horizontale dans un fluide visqueux. Dans les expériences de Ambari, Gauthier-Manuel et Guyon [2], la sphère était maintenue par un champ magnétique et déplacée à vitesse constante vers une paroi plane. Dans tous les cas, le mouvement de la sphère était mesuré visuellement avec un microscope. Les plus petites distances observées correspondaient à des distances sans dimension $\varepsilon$ (rapport entre la distance à la paroi et le rayon de la sphère) de l'ordre de $10^{-2}$. Les résultats expérimentaux trouvés étaient tous en bon accord avec la théorie des écoulements à faible nombre de Reynolds, ou théorie de la lubrification.

Plus récemment, Smart et Leighton [5] ont étudié le domaine des distances plus petites en mesurant le temps que met une particule sphérique en sédimentation pour arriver sur une paroi plane horizontale ou pour s'écarter d'une paroi plane horizontale située au-dessus. Ils ont montré que le comportement de la sphère s'écarte de la loi prévue par la théorie de la lubrification pour des distances sans-dimension $\varepsilon$ de l'ordre de $10^{-3}$; la force sur la particule dépend alors de sa rugosité.

1.3 DIFFÉRENCES PAR RAPPORT ÁUX EXPÉRIENCES ANTÉRIEURES. - Dans cet article, nous considérons une sphère èn sédimentation et observons directemént le mouvement de la sphère lorsqu'elle s'approche d'uñe paroi plane horizontale jusqu'à venir en contact avec celle-ci. Les déplacements sont mesurés avec une très bonne sensibilité de l'ord'ré de $5 \times 10^{-9} \mathrm{~m}$. Ceci est réalisé grâce à̀ une technique expérimentale basée sur l'interférómétrie, tèchnique dont l'emploi est à notre connaissance nouveau dans ce contexte.

Dans le chapitre 2 nous décrivons le principe de la technique interférométrique et le dispositif expérimental. Puis, dans le chapitre 3, nous exposons la procédure expérimentale et la méthode d'exploitation des résultats. Les résultats expérimentaux sont présentés et discutés dans le chapitre 4. Enfin la conclusion est dans le chapitre 5. 


\section{Principe de la mesure et dispositif expérimental.}

2.1 PRINCIPE DE LA TECHNIQUE INTERFÉROMÉTRIQUE. - La technique employée, sans être classique n'est pas nouvelle. Cependant, elle ne semble pas avoir été utilisée dans le présent contexte expérimental. Aussi, bien que l'objet de cet article soit plutôt d'illustrer les performances qu'elle permet d'atteindre, il nous paraît nécessaire d'en rappeler les idées directrices avant de décrire sa réalisation.

La technique «classique » de mesure interférométrique de déplacement utilise la variation temporelle d'un système d'interfërences (en général en teinte plate), obtenues à l'aide de réflecteurs de qualité optique : miroirs, réseaux (on remarque que ces objets peuvent être caractérisés par un spectre spatial de type «Dirac»). C'est le mouvement relatif des réflecteurs qui est mesuré. Les caractéristiques essentielles de la mesure sont :

1) la sensibilité (on mesure le rapport déplacement/longueur d'onde);

2) la précision, limitée par la connaissance de la longueur d'onde ;

3) la nécessité d'un réglage très précis de l'interféromètre, faute duquel on n'obtient aucun signal utilisable. L'emploi de réflecteurs de type dièdre déplace le besoin de précision du réglage de l'interféromètre à la fabrication des réflecteurs (ces notions classiques d'interférométrie sont décrites et analysées dans les manuels d'optique générale).

Cette dernière propriété rend l'interféromètre "classique " difficile ou impossible à utiliser dans bon nombre de situations expérimentales, soit par impossibilité de lier un réflecteur " optique » à l'objet en déplacement, soit en raison de difficultés à régler l'interféromètre, ou à conserver son réglage au cours du déplacement. Il est clair que ces remarques s'appliquent au contexte expérimental décrit dans cet article.

En réalité, il n'est nullement impossible de mesurer avec une sensibilité interférométrique le déplacement d'objets quelconques, c'est-à-dire à spectre spatial quelconque, à condition d'accepter des performances inférieures pour la précision de la mesure et l'efficacité énergétique. La technique est décrite et analysée en référence [4] dans le cas extrême des surfaces diffusantes et pour une configuration interférométrique particulière.

Pour en comprendre l'idée directrice, il suffit de remarquer que le caractère «à spectre spatial très étroit» des composants optiques est responsable des propriétés 2) et 3) mentionnées plus haut, mais non de la sensibilité, qui est liée uniquement à l'existence des interférences.

Dans ces conditions, on peut, lorsqu'un réflecteur à spectre large est utilisé, restaurer les conditions d'une mesure de précision quelconque en effectuant avant détection, sur les surfaces d'onde interférant, un filtrage passe-bande des fréquences spatiales. Le dispositif se comporte alors comme si l'objet réflecteur avait pour spectre spatial la bande sélectionnée, qui peut être très étroite. Autrement dit, on synthétise un réflecteur qui peut être (dans certaines limites déterminées par la diffraction) arbitrairement proche d'un miroir « optique ». L'intérêt majeur de cette procédure est que le système fournit toujours un signal exploitable tant que l'objet envoie de l'énergie dans la pupille de filtrage. En effet, comme le spectre de l'objet est large, il renvoie de la lumière dans un angle solide important, et le réglage de l'interféromètre est très peu critique : il peut donc être peu précis et peu stable au cours du déplacement.

Mais cette relative facilité de réglage est payée par une précision de mesure plus faible. Il est clair que le filtrage spatial rejette une grande part de l'énergie entrant dans l'interféromètre, ce qui peut engendrer des problèmes de rapport signal/bruit si la puissance du laser est trop faible. D'autre part, la précision de la mesure est limitée par la précision avec laquelle les paramètres d'alignement du montage sont connus, ainsi que par la largeur de bande du filtrage spatial. Par conséquent, toute imprécision ou dérive du réglage affecte la précision de 
la mesure (mais non sa sensibilité, d'après ce qui précède). Une analyse plus détaillée sortirait du cadre de cet article.

On remarque que nous n'avons jamais fait d'hypothèses sur le dispositif interférométrique (sinon l'hypothèse implicite qu'il s'agit d'un système à deux ondes). En effet les principes qui viennent d'être décrits restent valables quel que soit l'interféromètre employé. Le paragraphe suivant décrit leur mise en œuvre pratique, compte-tenu des mesures à réaliser.

2.2 DisPoSITIF EXPÉRIMENTAL ET PROCÉDURE DE RÉGLAGE. - Le dispositif expérimental est composé de deux parties essentielles. La première partie est consacrée au montage optique et mécanique, la deuxième partie traite de l'électronique d'acquisition et de traitement des signaux. Le schéma de liaison entre les deux parties, ainsi que la désignation des divers éléments constituant le dispositif sont présentés sur la figure 1.

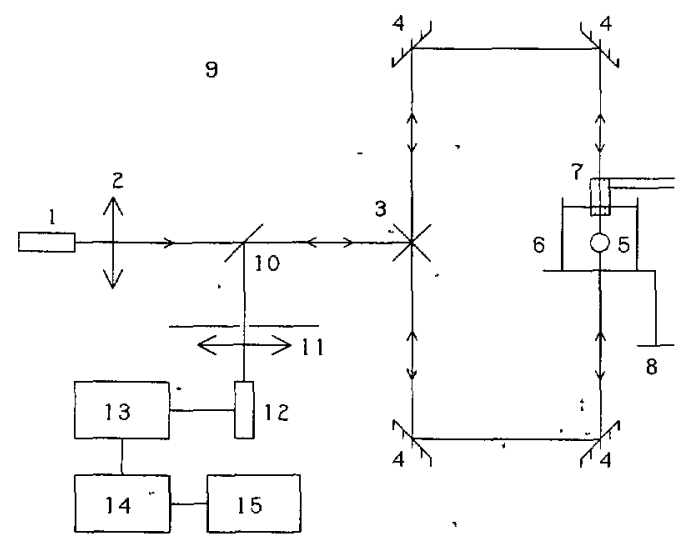

Fig. 1. - Dispositif expérimental. 1) Laser He-Ne ; 2) Lentille de focalisation ; 3) Prisme séparateur de faisceaux ; 4) Miroirs ; 5) Sphère ; 6) Cuve cylindrique contenant de l'huile Rhodorsyl 47V100000 ; 7) Electro-aimant, avec fenêtre optique plóngeant dans le liquide ; 8) Support $X Y Z$ (mise en station) ; 9) Table anti-vibratoire ; 10) Séparatrice; 11) Lentille d'observation ; 12) Viseur optique ; 13) Photomultiplicateur, amplificateur et filtre passe bas ; 14) Oscilloscope ; 15) Calculateur (IBM PC/AT) muni de la carte de conversion analogique-digital.

[Experimental setup. 1) He-Ne laser ; 2) Focusing lens ; 3) Beam splitter ; 4) Mirrors ; 5) Sphere ; 6) Cylindrical vessel containing Rhodorsyl oil of type $47 \mathrm{~V} 100000$; 7) Electro-magnet with optical window immersed in the liquid ; 8) XYZ stand ; 9) Vibrationless table ; 10) Beam splitter ; 11) Observation lens ; 12) Optical viewfinder; 13) Photomultiplier, amplifier and low pass filter ; 14) Oscilloscope ; 15) IBM PC/AT microcomputer with the add-in board for analog-digital conversion.]

2.2.1 Optique. - Nous disposions d'un interféromètre à trajets symétriques, comportant la possibilité de régler les directions des faisceaux sortants. Dans ces conditions, il était commode d'utiliser cet interféromètre selon le schéma de la figure 1, où l'on voit que la bille est à la fois éclairée et observée par chaque voie de l'interféromètre, sur deux calottes diamétralement opposées. De plus, il est clair que pour pouvoir suivre la chute sur une distance importante $(1$ à $2 \mathrm{~cm}$ ) sans perte de réglage, l'axe commun des faisceaux doit être vertical. Avec un tel dispositif, le signal détecté suit une loi en $\sin [2 \pi \Delta x(4 n / \lambda)], n$ étant l'indice du milieu dans lequel se déplace la bille, $\Delta x$ son déplacement et $\lambda$ la longueur d'onde dans le vide. De plus, l'interféromètre fonctionne au voisinage de la différence de marche nulle. 
Le laser (de puissance $15 \mathrm{~mW}$ ) produit donc un faisceau, qui, après focalisation, est séparé en deux faisceaux de puissances à peu près égales. Les faisceaux sont ensuite dirigés sur la bille selon deux axes de propagation confondus et opposés. On peut estimer qu'ils éclairent chacun une zone de l'ordre de $0,5 \mathrm{~mm}$ de diamètre. A l'aide d'une lentille fortement diaphragmée, on forme à travers l'interféromètre une image fortement filtrée de chaque calotte éclairée.

On détecte les interférences produites par superposition des images des zones éclairées. Une configuration optimale consiste à régler le filtrage de façon à ce que la zone éclairée soit à peu près égale à une tache de résolution. La dimension optimale du détecteur est alors de l'ordre de la moitié de celle de la tache de diffraction image.

L'essentiel de la procédure de réglage consiste à assurer (au niveau de la bille) la verticalité et la confusion des axes optiques de chaque voie de l'interféromètre. La verticalité peut être obtenue pour le faisceau descendant avec une précision suffisante par autocollimation sur un bain de mercure. La confusion des faisceaux est réalisée lorsqu'une teinte quasi plate (c'est-àdire un état d'interférence à peu près uniforme) est réalisée sur la surface du détecteur. Le montage donne lạ possibilité d'obțenir ce réglage avant le lâcher de la bille (c'est-à-dire à plusieurs centimètres de la position finale), tout en garantissant la qualité du réglage jusqu'à la fin de l'expérience. Dans ces conditions, le laser utilisé permettant une différence de marche de quelques centimètres, la chute de la bille peut être suivie entièrement, même si l'on ne mesure que l'approche de la paroi.

La sphère (5) se déplace verticalement dans l'huile (Rhodorsil 47V100000, de viscosité cinématique $\nu=0,1 \mathrm{~m}^{2} / \mathrm{s}$ ) contenue dans une cuve cylindrique (6) de diamètre $0,05 \mathrm{~m}$ et dé hauteur 0,06 $\mathrm{m}$ posée sur un support $X Y Z$.

- Sur ce support est fixé un électro-aimant (7) dont le rôle est double :

1) maintenir la bille avant le lâcher, de façon à permettre le réglage initial de l'interféromètre avec la bille ;

2) assurer un lâcher de la bille sans impulsion latérale; à cette fin, il est alimenté en courant alternatif de façon à éviter, après coupure de l'alimentation, une aimantation rémanente dont la dissymétrie éventuelle pourrait faire dévier la bille de la verticale prévue.

Par ailleurs, le faisceau supérieur, qui voyage d'abord dans l'air, doit rentrer dans l'huile et donc traverser une interface. Il est clair (et nous avons constaté) qu'un dioptre air-huile est déformé par la mise en chute de la bille, même si celle-ci est immergée au départ. D'où le dispositif de la figure 2, qui comporte une fenêtre optique dont la face inférieure est immergée et la face supérieure est dans l'air.

Les sphères utilisées ont $2 \times 10^{-3} \mathrm{~m}$ et $2,5 \times 10^{-3} \mathrm{~m}$ de rayon. La rugosité des billes, observée au microscope électronique à balayage est de l'ordre de $1,5 \times 10^{-6} \mathrm{~m}$ tel que le montre la photo de la figure 3.

Les nombres de Reynolds pour les sphères en chute libre sans effet de paroi sont de $2 \times 10^{-5}$ et $4 \times 10^{-5}$

2.2.2 Acquisition et traitement des signaux. - Le détecteur est une fibre optique de diamètre $150 \mu \mathrm{m}$, couplée à un photomultiplicateur. Le signal électrique (courant faible sous haute impédance) est converti par un amplificateur suiveur en un signal de tension basse impédance, et soumis en même têmps à un filtrage passe bas. Bien entendu, on règle la fréquence de coupure à une valeur assez haute pour que le signal de déplacement (qui est pratiquement une sinusoïde pure) ne subisse qu'un déphasage négligeable.

En ce qui concerne l'acquisition, nous disposons d'un micro-ordinateur IBM de type PC/AT (80286) fonctionnant à $6 \mathrm{MHz}$ avec $512 \mathrm{Ko}$ de RAM. Dans l'unité centrale est implantée une carte d'acquisition et de conversion analogique-digitale. 


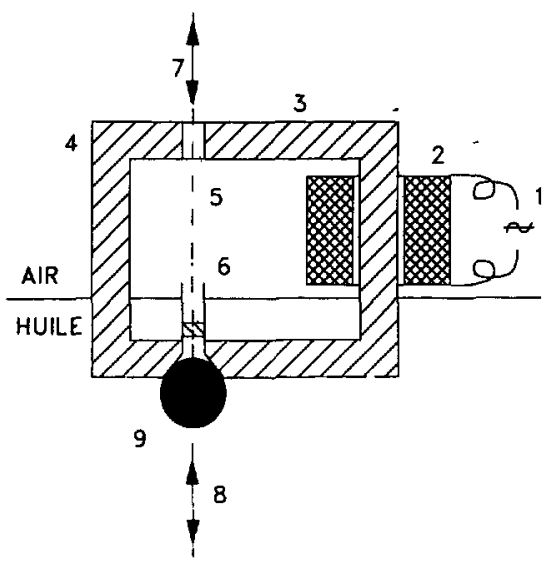

Fig. 2. - Schéma de fonctionnement de l'électro-aimant (vue en coupe). 1) Alimentation en courant alternatif ; 2) Bobine ; 3), 4) Circuit magnétique (acier doux); 5) Ouverture pour le passage du faisceau ; 6) Fenêtre optique ; 7), 8) Direction des faisceaux incidents et réfléchis ; 9) Sphère avant lâcher.

[Sketch of the device for releasing the sphere (section view). 1) AC supply ; 2) Coil ; 3), 4) Magnetic circuit (armco alloy) ; 5) Opening for the crossing beam ; 6) Optical window ; 7), 8) Direction of the incident and reflected beams; 9) Sphere before release.]

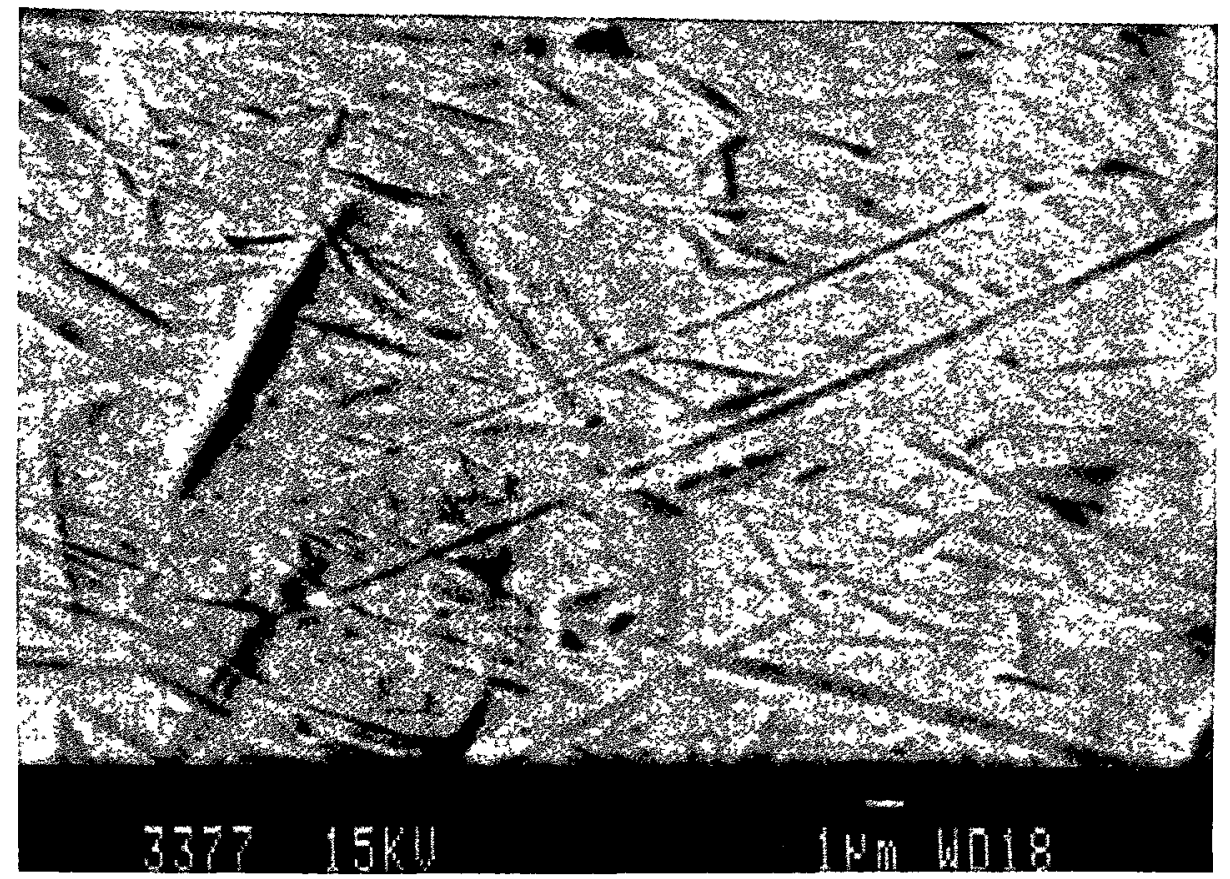

Fig. 3. - Etat de surface d'une sphère en acier doux observée au microscope électronique à balayage. La profondeur des rugosités est estimée à $1,5 \times 10^{-6} \mathrm{~m}$.

[Scanning electron micrograph showing the surface condition of a mild steel sphere. The depth of rugosities is estimated to be $1.5 \times 10^{-6} \mathrm{~m}$.] 
L'utilisation de la carte se fait par l'intermédiaire de primitives réalisées en langage Machine interfacé en langage Basic.

Dans ces conditions, la fréquence d'acquisition maximum est $40 \mathrm{kHz}$.

\section{Procédure expérimentale et méthode d'exploitation des résultats.}

L'interféromètre, y compris la bille immobile en position de départ, est d'abord réglé pour obtenir un signal optimal (cf. Sects. précédentes). On utilise pour cela un oscilloscope qui visualise le signal d'interférence, et on met le montage en vibration en donnant de légers chocs à la table d'expérience. Cet oscilloscope permet également de contrôler la qualité du signal au cours de l'expérience, et de détecter la fin du mouvement de la bille.

Une fois que différents essais sont jugés satisfaisants, nous procédons à l'acquisition et au stockage des signaux par la carte. Cette acquisition et ce stockage se font en temps réel, ce qui crée une contrainte supplémentaire quant au suivi sur une longue période du mouvement. Comme c'est surtout la zone où le contact sphère-plan a lieu qui nous intéresse, nous avons décidé de ne stocker que les informations relatives à cette zone, dans la limite de la place disponible en mémoire vive ( $512 \mathrm{Ko}$ de RAM). Dans un premier temps, on détermine le temps nécessaire à la sphère pour parcourir la distance qui sépare sa position initiale fixe (sur l'électro-aimant) du fond de la cuve (la fin du mouvement est décelée par une visualisation directe sur l'oscilloscope). Puis en fonction de la fréquence horloge du calculateur, de sa place mémoire et de l'instant prévu pour la fin du mouvement, nous déclenchons l'acquisition du signal par un programme couplé avec l'interrupteur de l'électro-aimant.

Les données sont ensuite mémorisées sur un support amovible pour être traitées ultérieurement. Un programme permet de reproduire ce signal et de tracer la courbe de variation en fonction du temps. Des exemples des franges observées à des temps successifs sont donnés sur les figures $4,5,6$ et 7. Pour toutes ces figures, la fréquence d'acquisition des données est $2,4 \mathrm{kHz}$ et l'intervalle de temps de visualisation représenté suivant l'abscisse est $4,2 \times 10^{-2} \mathrm{~s}$.

Ces figures sont les divers échantillons de signaux produits par le mouvement d'une sphère de rayon $2 \times 10^{-3} \mathrm{~m}$ dans l'huile à des intervalles de temps donnés au cours de l'acquisition. La tension à l'entrée de la carte est contrôlée et maintenue fixe pendant toute l'opération. On procède ensuite à l'échantillonnage des signaux par une boucle sur la fréquence d'acquisition ; ceci, afin d'obtenir une bonne reproduction du signal stocké. Par ce processus, nous avons constaté qu'une fréquence d'acquisition de $2400 \mathrm{~Hz}$ dans une zone où le contact sphère-plan a lieu permet d'obtenir cette bonne reproduction.

Dans l'exemple donné sur les figures 4, 5, 6 et 7, on suit l'évolution temporelle du signal depuis le début de l'acquisition jusqu'à sa fin qui correspond à la fin du mouvement. Cette fin est visible sur la figure 7 , au temps $t=11 \mathrm{~s}$, où le signal est représenté par une droite horizontale.

Le signal représenté par ces courbes n'a subi d'autre filtrage que le filtrage passe bas avant acquisition. Aucune transformation numérique n'a été appliquée après l'acquisition. Les courbes sont donc la reproduction exacte des signaux stockés. Du fait de leur faible niveau de bruit, il est clair que la résolution qu'elles permettent sur le déplacement est excellente, probablement de l'ordre du vingtième d'interfrange, soit $\lambda / 80 n=5,2 \mathrm{~nm}$. Par ailleurs, la vitesse d'évolution du phénomène étudié (vitesse de chute) est faible (cf. paragraphe suivant), et il suffit amplement de considérer un échantillonnage au quart de frange $(\lambda / 16 n=$ $26 \mathrm{~nm}$ ). Nous discuterons la précision des mesures dans le chapitre 4.

Le traitement consiste donc simplement à décompter les extrema et les passages par le niveau moyen, à partir du point d'arrêt de la sphère. Nous obtenons ainsi la distance $z_{\mathrm{p}}$ entre le centre de la sphère et la paroi. 
Tension (Volt)

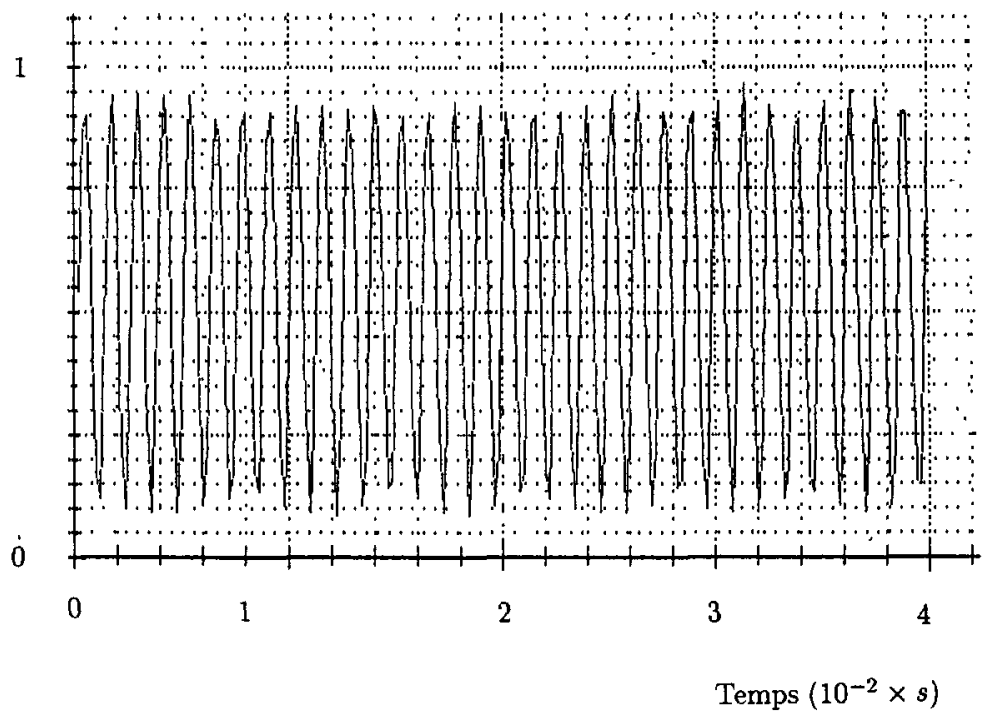

Fig. 4. - Visualisation du signal (tension en fonction du temps) au début de l'acquisition de données, soit à $5 \times 10^{-4} \mathrm{~m}$ de la paroi.

[Plot of the signal (voltage versus time) at the beginning of the data acquisition, that is at a distance $5 \times 10^{-4} \mathrm{~m}$ away from the. wall.]

Tension (Volt)

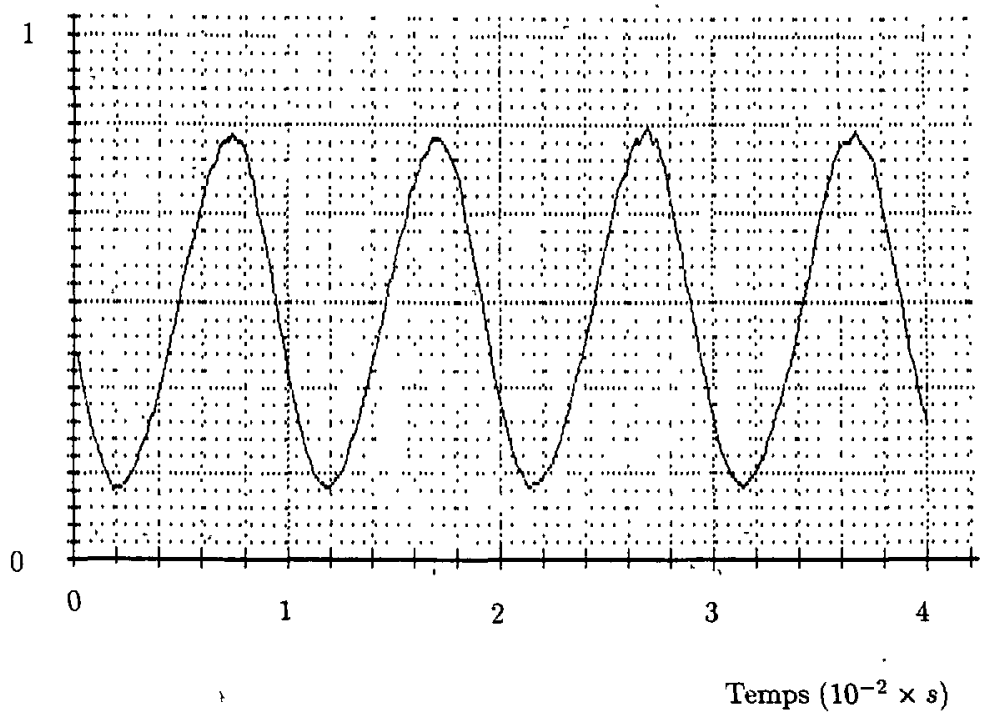

Fig. 5. - Visualisation du signal après $4 \mathrm{~s}$.

[Plot of the signal after $4 \mathrm{s.]}$ 


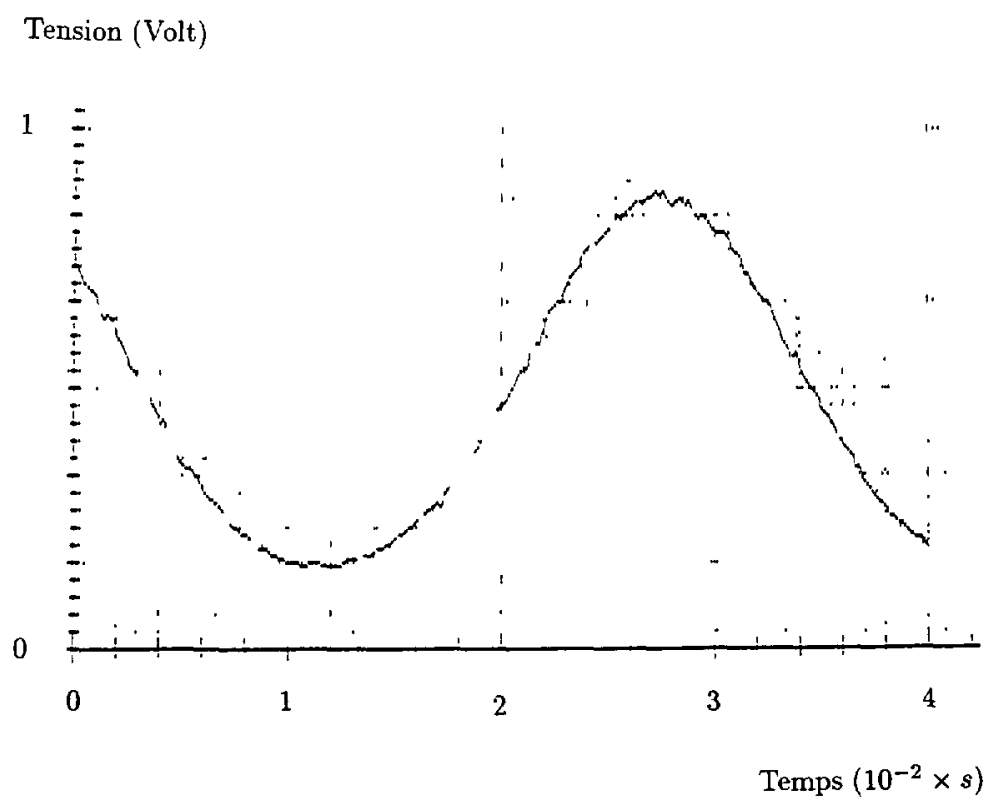

Fig. 6. - Visualisation du signal après $8 \mathrm{~s}$.

[Plot of the signal after $8 \mathrm{~s}$.]

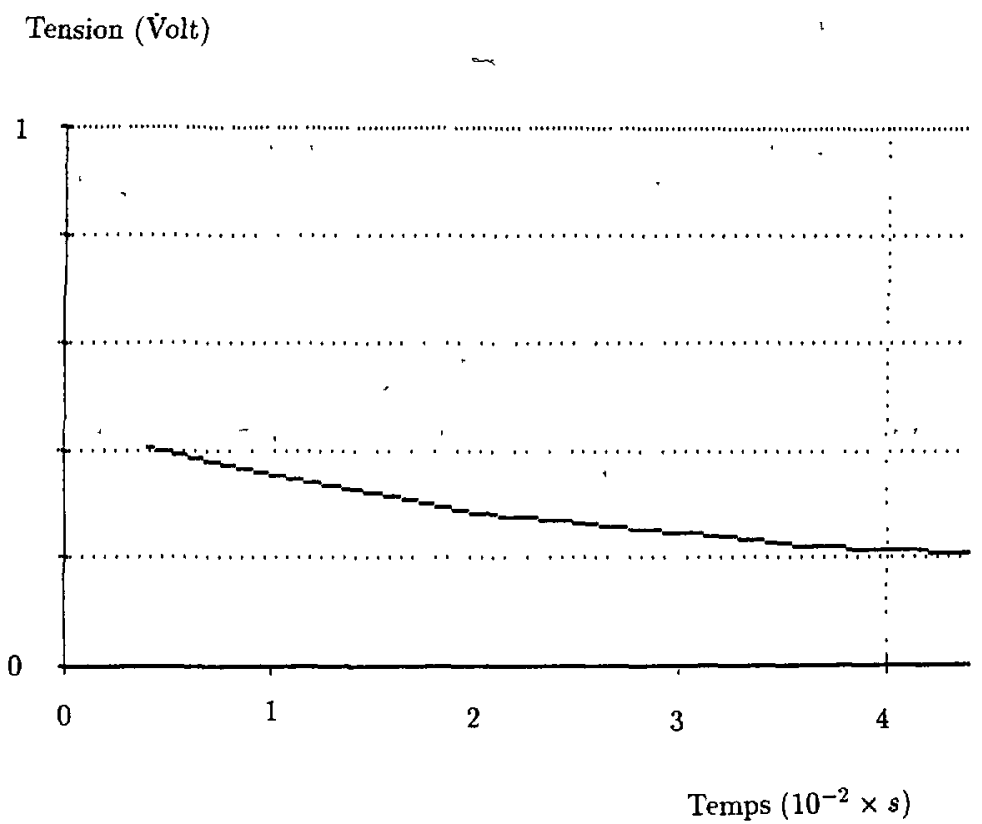

Fig. 7. - Visualisatión du signal après $11 \mathrm{~s}$.

[Plot of the signal after $11 \mathrm{~s}$.] 
Connaissant la position $z_{\mathrm{p}}$ de la sphère au cours du temps, nous obtenons sa vitesse $v_{\mathrm{p}}$ par dérivation par rapport au temps. Pour ce faire, nous déterminons le déplacement de la sphère sur des intervalles de temps égaux à $0,25 \mathrm{~s}$.

Nous pouvons ensuite en déduire l'accélération de la sphère, puis contrôler que le mouvement est quasi stationnaire et, finalement, en déduire un coefficient de frottement que nous allons définir maintenant.

Rappelons d'abord la formule bien connue de Stokes [6] donnant la force de traînée s'exerçant sur une sphère solide se déplaçant à vitesse constante $v_{\mathrm{ps}}$ dans un fluide illimité au repos :

$$
F=-6 \pi a \mu v_{\mathrm{ps}}
$$

où $a$ est le rayon de la sphère et $\mu$ la viscosité dynamique du fluide. Cette formule est valable dans le cadre de l'hypothèse d'un petit nombre de Reynolds:

$$
\mathrm{Re}=\frac{a v_{\mathrm{ps}} \rho}{\mu} \ll 1
$$

où $\rho$ est la masse volumique du fluide.

Pour une sphère solide se déplaçant à vitesse constante $v_{\mathrm{p}}$ perpendiculairement à une paroi solide au repos dans un fluide qui est au repos à l'infini, l'analyse dimensionnelle montre que dans le cadre de l'hypothèse (2), la force de traînée sur la sphère est de la forme :

$$
F=-6 \pi a \mu f_{z z}^{\mathrm{T}} v_{\mathrm{p}}
$$

où $f_{z z}^{\mathrm{T}}$, coefficient de frottement, n'est fonction que de $z_{\mathrm{p}} / a$. Notons que d'après (1), $f_{z z}^{\mathrm{T}} \rightarrow 1$ lorsque $z_{\mathrm{p}} / a \rightarrow \infty$.

Lorsque la sphère tombe dans un fluide visqueux au repos en direction d'une paroi plane horizontale, le mouvement de la sphère est instationnaire et on peut se demander si la formule stationnaire (3) est encore valable.

Dans le cas où la sphère est à une distance du plan telle que $z_{\mathrm{p}}-a$ est d'ordre $a$, $f_{z z}^{\mathrm{T}}$ est d'ordre unité et l'on peut montrer que la formule stationnaire (3) reste valable. En effet, le temps caractéristique de mise en vitesse de l'écoulement est d'après les équations de Navier-Stokes :

$$
\tau_{\nu}=\frac{a^{2}}{\nu}
$$

où $\nu$ est la viscosité cinématique du fluide. Le temps que met la sphère pour parcourir à vitesse $v_{\mathrm{ps}}$ une distance de l'ordre de grandeur de son rayon est

$$
\tau=\frac{a}{v_{\mathrm{ps}}}
$$

On peut alors remarquer que

$$
\frac{\tau_{\nu}}{\tau}=\operatorname{Re} \ll 1
$$

c'est-à-dire qu'à l'échelle de temps $\tau$, l'écoulement est quasi stationnaire.

Lorsque la sphère s'approche très près du plan à vitesse constante l'utilisation de la théorie de la lubrification permet de montrer que

$$
f_{z z}^{\mathrm{T}}=\frac{1}{\varepsilon}
$$


où

$$
\varepsilon=\frac{z_{\mathrm{p}}-a}{a} \ll 1
$$

est l'intervalle sans dimension entre la sphère et le plan. Dans le cas instationnaire, l'étude précise a été faite par Cox et Brenner [3] qui montrent que la formule (7) reste valable au premier ordre.

Nous exploiterons alors les résultats expérimentaux en supposant que la formule de type (3) de type stationnaire reste valable dans le mouvement de chute libre. Ecrivons alors l'équation du mouvement de la sphère :

$$
\frac{4}{3} \pi a^{3} \rho_{\mathrm{p}} \frac{\mathrm{d} v_{\mathrm{p}}}{\mathrm{d} t}=-6 \pi a \mu f_{z z}^{\mathrm{T}} v_{\mathrm{p}}+\frac{4}{3} \pi a^{3}\left(\rho_{\mathrm{p}}-\rho\right) g
$$

où $\rho_{\mathrm{p}}$ est la masse volumique de la sphère et $g$ l'accélération de la gravité. Connaissant l'évolution de la vitesse expérimentale au cours du temps, nous pouvons en déduire l'accélération $\frac{\mathrm{d} v_{\mathrm{p}}}{\mathrm{d} t}$ puis le coefficient de frottement par (9).

En fait, le terme d'accélération dans (9) est négligeable. En effet, d'après (9), le temps caractéristique d'accélération de la sphère est

$$
\tau_{\mathrm{p}}=\frac{\frac{4}{3} \pi a^{3} \rho_{\mathrm{p}}}{6 \pi a \mu f_{z z}^{\mathrm{T}}}=\frac{2}{9} \frac{a^{2}}{\nu} \frac{\rho_{\mathrm{p}}}{\rho} \frac{1}{f_{z z}^{\mathrm{T}}}
$$

A distance du plan telle que $\frac{z_{\mathrm{p}}-a}{a}=O(1)$, alors $f_{z z}^{\mathrm{T}}=O(1)$, et comme $\rho_{\mathrm{p}} / \rho=O(1)$ pour un liquide, alors $\tau_{\mathrm{p}} \sim \tau_{\nu}$. D'où

$$
\frac{\tau_{\mathrm{p}}}{\tau} \sim \operatorname{Re} \ll 1
$$

Le temps de mise en vitesse de la sphère est négligeable.

A une distance de la paroi telle que (8) est vérifiée, (7) s'applique et comme $\rho_{\mathrm{p}} / \rho=O(1)$ pour un liquide, alors $\tau_{\mathrm{p}} \sim \tau_{\nu} \varepsilon$. Le temps caractéristique de déplacement de la sphère sur une distance de l'ordre de $\varepsilon a$ est, en supposant le mouvement quasi stationnaire :

$$
\tau=\varepsilon a\left(\frac{4 / 3 \pi a^{3}\left(\rho_{\mathrm{p}}-\rho\right) g \varepsilon}{6 \pi a \mu}\right)^{-1}=\frac{a}{v_{\mathrm{ps}}}
$$

On a alors

$$
\frac{\tau_{\mathrm{p}}}{\tau}=\varepsilon \operatorname{Re} \ll 1
$$

et l'on démontre ainsi que le mouvement de la sphère est bien quasi stationnaire.

L'accélération de la sphère étant négligeable, le coefficient de frottement est alors donné par la formule plus simple:

$$
f_{z z}^{\mathrm{T}}=\frac{v_{\mathrm{ps}}}{v_{\mathrm{p}}}
$$

En exploitant les résultats expérimentaux, nous vérifions effectivement que l'accélération de la sphère est négligeable et obtenons alors $f_{z z}^{\mathrm{T}}$ par la formule (14). 


\section{Résultats expérimentaux et discussion.}

On a représenté figures 8 et 9 le coefficient de frottement obtenu à partir des mesures expérimentales pour des billes de rayons $2 \times 10^{-3} \mathrm{~m}$ et $2,5 \times 10^{-3} \mathrm{~m}$ en fonction des valeurs de $\varepsilon$ obtenues au cours de la sédimentation. Les coordonnées sont logarithmiques èt on a représenté en trait plein la droite correspondant à la valeur théorique (7) du coefficient de frottement.

On voit sur ces figures que la comparaison théorie-expérience est bonne pour $\varepsilon \geqslant 10^{-2}$ ce qui correspond à une séparation $\varepsilon a \geqslant 2 \times 10^{-5} \mathrm{~m}$. Les valeurs expérimentales du coefficient de frottement sont inférieures aux valeurs théoriques pour des valeurs de $\varepsilon$ dont l'ordre de grandeur est au plus $10^{-3}$, ce qui correspond à $\varepsilon a \leqslant 2 \times 10^{-6} \mathrm{~m}$. Remarquons que l'étude de la surface par microscopie électronique à balayage (cf. Chap. 2) avait montré que la rugosité des billes est de cet ordre de grandeur. Il est ainsi clair que la diminution observée du coefficient de frottement est due aux effets de' rugosité.

A ce point, il est utile de bien préciser la définition de la distance à la paroi $\varepsilon a$. D'après la procédure expérimentale utilisée (cf. Chap. 3), $\varepsilon=0$ lorsque la bille est immobile et l'on estime alors qu'elle est en contact avec la paroi. $\varepsilon \vec{a}$ correspond donc à la distance entre la paroi plane et l'enveloppe extérieure des rugosités. Cette sphère enveloppe des rugosités est en quelque sorte poreuse et il est physiquement compréhensible que son coefficient de frottement soit inférieur à celui d'une sphère pleine. En effet, le coefficient de frottement d'une sphère pleine croît comme $1 / \varepsilon$ parce que le fluide situé dans la couche de lubrification, entre la sphère et la paroi, peut difficilement s'extraire de cette couche lorsque la sphère s'approche de la paroi. Dans le cas d'une sphère poreuse, le fluide a la possibilité supplémentaire de s'échapper par l'intérieur de la sphère.

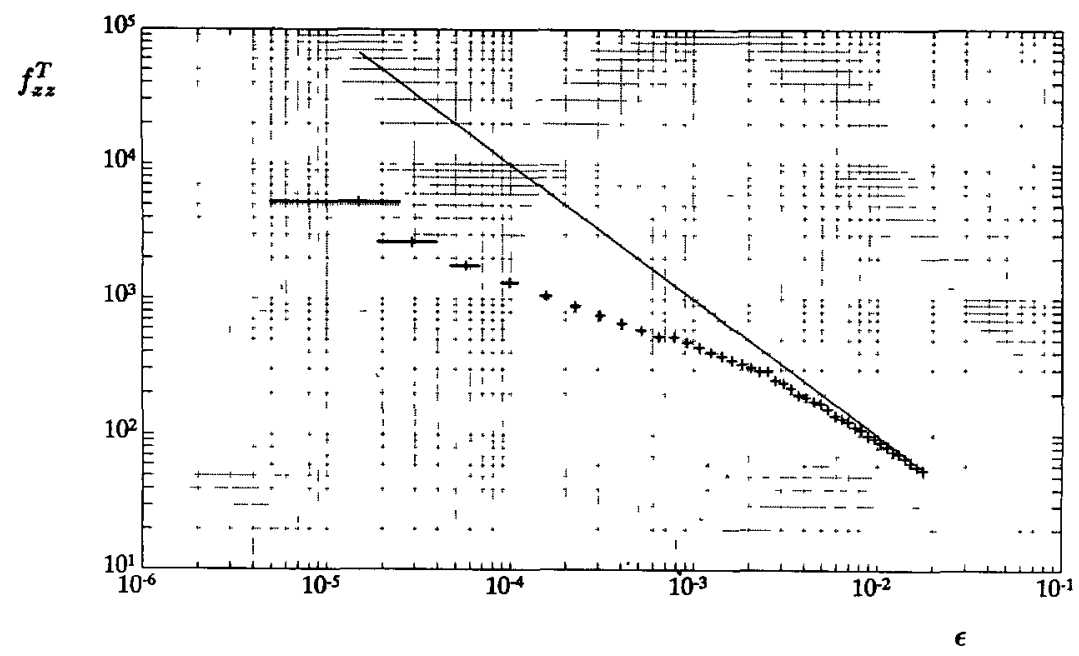

Fig. 8. - Coefficient de traînée $f_{z z}^{\mathrm{T}}$ en fonction de la distance réduite à la paroi $\varepsilon$ pour une bille de $2 \times 10^{-3} \mathrm{~m}$ de rayon: comparaison théorie-expérience. L'incertitude sur $\varepsilon$ est estimée à $10^{-5}$ (cf. la discussion dans le paragraphe 4).

[Friction coefficient $f_{z z}^{\mathrm{T}}$ versus the nondimensional gap $\varepsilon$ for a ball with radius $2 \times 10^{-3} \mathrm{~m}$ : comparison between theory and experiment. The experimental error on $\varepsilon$ is estimated to be $10^{-5}$ (cf. discussion in Sect. 4).] 


\section{W.URE PAR INTERFÉROMETRIE LASER}

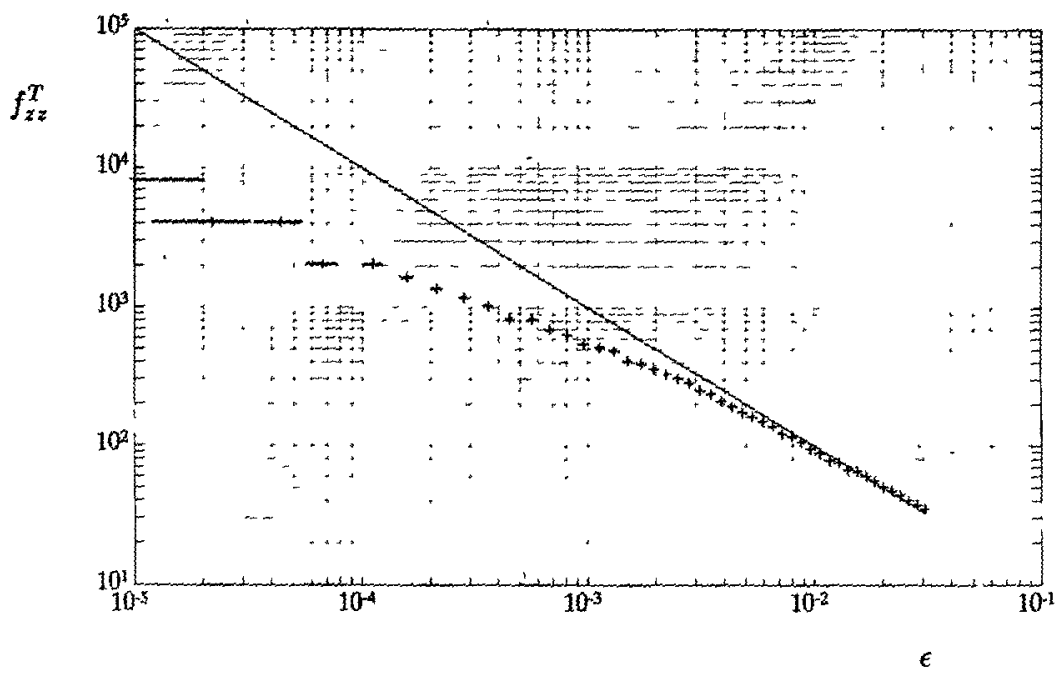

Fig. 9. - Coefficient de trainè $f_{z z}^{\mathrm{T}}$ en fonction de la distance réduite à la paroi $\varepsilon$ pour une bille de $2,5 \times 10^{-3} \mathrm{~m}$ de rayon : comparaison théorie-expérience. L'incertitude sur $\varepsilon$ est estimé a $10^{-5}$ (cf. la discussion dans le Chap. 4).

[Friction coefficient $f_{z=}^{T}$ versus the nondimensional gap $\varepsilon$ for a ball with radius $2.5 \times 10^{-3} \mathrm{~m}$ : comparison between theory and experiment: The experimental error on $\varepsilon$ is estimated to be $10^{-5}$ (cf. the discussion in Sect. 4).]

Les effets de rugosité ne se manifestent qu'à courte portée, car on a vu que pour $\varepsilon=10^{-2}$ (soit environ 10 fois la rugositè), la sphère se comporte comme si elle était lisse.

Enfin, il apparaît que la technique de mesure permet d'obtenir des résultats jusqu'à des valeurs $\varepsilon=10^{-5}$ ce qui est très inférieur à l'échelle de la rugosité.

Un autre phénomène responsable de lécart entre la théorie et l'expérience pourrait être le comportement non newtonien de l'huile dù aux grands gradients de vitesse qui apparaissent entre la sphère et la paroi lorsque l'intervalle entre les deux devient très petit. Cet effet peut sévaluer en utilisant la théorie de la lubrification et les données techniques sur l'huile Rhodorsyl utilisée.

Considérons un système de coordonnées cylindriques (Fig. 10) avec l'axe z suivant laxe de

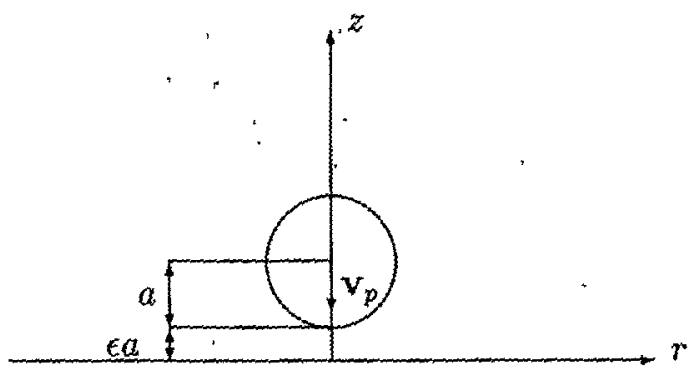

10. - Système de coordonnìes cylindriques pour une sphère de rayon $a$ située à une distance un plan. La sphère se déplace vers le plan avec une vitesse $v_{p}$.

$\mathrm{n}$ of cylindrical coordinates for a sphere with radius $a$ at a distance $z a$ from a wall. The sphere towards the wall with a velocity $\mathrm{v}_{\mathrm{p}}$.] 
symétrie de la figure et $z=0$ représentant la paroi. En utilisant l'équation de continuité du fluide, la vitesse moyenne du fluide expulsé de l'intervalle entre la sphère et la paroi est :-

$$
\bar{u}=\frac{r}{2 Z} v_{p},
$$

où $Z$ est la distance entre la sphère et la paroi au point $r$. En résolvant l'équation de quantité de mouvement du fluide, on trouve que la vitesse du fluide a un profil parabolique entre la sphère et la paroi :

$$
u=\frac{6 \bar{u} z(Z-z)}{Z^{2}}
$$

Les gradients de vitesse les plus importants se trouvent à la paroi et sur la sphère. Ils ont pour valeur :

$$
\frac{6 \bar{u}}{Z}=\frac{3 r}{Z^{2}} v_{\mathrm{p}}
$$

La valeur de $Z$ peut s'obtenir par développement pour $\varepsilon$ petit au voisinage de l'origine des coordonnées :

$$
Z=\frac{r^{2}}{2 a}+\varepsilon a+O\left(\varepsilon^{2}\right)
$$

En reportant cette valeur dans l'expression (17) du gradient de vitesse, on trouve que la fonction de $r$ ainsi obtenue est maximum pour

$$
r=\sqrt{\frac{2 \varepsilon}{3}} a .
$$

La valeur maximum $u_{\mathrm{m}}^{\prime}$ du gradient de vitesse est alors :

$$
u_{\mathrm{m}}^{\prime}=\frac{9 \sqrt{6}}{16} \varepsilon^{-3 / 2} \frac{v_{\mathrm{p}}}{a}
$$

Compte tenu de (14), nous pouvons aussi écrire ce résultat sous la forme :

$$
u_{\mathrm{m}}^{\prime}=\frac{9 \sqrt{6}}{16} \varepsilon^{-1 / 2} \frac{v_{\mathrm{ps}}}{a}
$$

Nous avons donc montré que, pour une sphère donnée à une distance donnée de la paroi, le gradient de vitesse du fluide est maximum sur deux cercles situés respectivement sur la sphère et sur la paroi. Ces cercles sont centrés sur l'axe $z$ et leur rayon est donné par (19). La valeur maximum du gradient de vitesse est donnée par (21).

Ainsi par exemple, pour la bille de rayon $a=2 \times 10^{-3} \mathrm{~m}$, la vitesse de chute mesurée sans effet de parois est : $v_{\mathrm{ps}}=5,93 \times 10^{-4} \mathrm{~m} / \mathrm{s}$. Pour une distance à la paroi telle que $\varepsilon=10^{-4}$, on obtient $u_{\mathrm{m}}^{\prime}=40,85 \mathrm{~s}^{-1}$ et pour $\varepsilon=10^{-5}$, on obtient $u_{\mathrm{m}}^{\prime}=51,03 \mathrm{~s}^{-1}$ Pour la bille de rayon $a=2,5 \times 10^{-3} \mathrm{~m}$, la vitesse de chute mesurée sans effet de parois est: $v_{\mathrm{ps}}=9,26 \times 10^{-4} \mathrm{~m} / \mathrm{s}$. Pour une distance à la paroi telle que $\varepsilon=10^{-4}$, on obtient $u_{\mathrm{m}}^{\prime}=129,19 \mathrm{~s}^{-1}$ et pour $\varepsilon=10^{-5}$, on obtient $u_{\mathrm{m}}^{\prime}=161,39 \mathrm{~s}^{-1}$.

D'après les données techniques sur l'huile Rhodorsyl 47V100000, la viscosité est constante jusqu'à un gradient de vitesse de $30 \mathrm{~s}^{-1}$. Elle décroît ensuite : la viscosité cinématique est de l'ordre de $0,09 \mathrm{~m}^{2} / \mathrm{s}$ pour un gradient de vitesse de $100 \mathrm{~s}^{-1}$ et de l'ordre de $0,08 \mathrm{~m}^{2} / \mathrm{s}$ pour $200 \mathrm{~s}^{-1}$ 
Compte tenu des gradients de vitesse calculés, la diminution de viscosité pour le cas d'une bille de rayon $a=2 \times 10^{-3} \mathrm{~m}$ est donc dans tous les cas inférieure à $5 \%$; pour une bille de rayon $a=2,5 \times 10^{-3} \mathrm{~m}$, elle est dans tous les cas inférieure à $13 \%$. Or, le coefficient de frottement diminue d'un facteur 10 lorsque la bille s'approche de la paroi, pour les deux rayons considérés. La variation de la viscosité due à l'apparition du comportement non newtonien n'apporte donc qu'une faible contribution à ce phénomène.

Pour conclure cette discussion, des théories hydrodynamiques incluant éventuellement différents phénomènes physiques restent à faire pour expliquer le comportement du coefficient de frottement aux faibles valeurs de $\varepsilon$.

En ce qui concerne la précision des mesures, nous estimons que l'interfrange est certainement connue à mieux que $1 \%$ près (réglage de l'interféromètre et bande passante du filtre spatial). Il en est donc de même, au mieux, pour la vitesse calculée à partir des déplacements. Cependant, pour les points correspondant aux points les plus proches de la paroi (jusqu'à $10^{-4}$ de proximité réduite), la vitesse est très faible et correspond à un changement d'état d'interférence de $1 / 3$ de frange (cas le plus défavorable). En admettant l'incertitude donnée plus haut, l'incertitude relative sur la vitesse est de l'ordre de $\pm 15 \%$; ceci correspond à l'augmentation de la dispersion des points expérimentaux sur la courbe des figures 8 et 9 , entre $10^{-4}$ et $10^{-5}$ de proximité réduite.

Par ailleurs, la précision de l'expérience est limitée par deux éléments :

1) La détermination du point d'arrêt d'après les courbes de déplacement, qui est sujette à une incertitude non négligeable. En effet, le point d'arrêt n'est pas (à première vue) un point de discontinuité, et la forme sinusoïdale de la fonction signal-déplacement peut dans certains cas accroître l'incertitude. Cette détermination peut être améliorée par une analyse fine du signal d'interférence. On admettra ici que l'erreur sur la détermination du point d'arrêt est de l'ordre du quart de frange. Il s'ensuit une incertitude sur $\varepsilon$ de l'ordre de $10^{-5}$. D'autre part, à son point d'arrêt, la bille peut encore être séparée de la paroi par une couche de liquide de l'ordre de quelques molécules. Il est clair que cette distance résiduelle bille-paroi ne peut pas être déterminée par la présente expérience. Supposons que cette distance soit de l'ordre de $10^{-8} \mathrm{~m}$; alors l'erreur systematique faite en supposant que le point d'arrêt est le point de contact avec la paroi est de l'ordre de $10^{-5}$ sur $\varepsilon$. L'erreur globale sur $\varepsilon$, somme des erreurs précédentes, se traduit en coordonnées $\varepsilon$ linéaires, par une simple translation de la fonction $f_{z z}^{\mathrm{T}}(\varepsilon)$ parallèlement à l'axe $\varepsilon$. En coordonnées $\log \varepsilon$, la même erreur se traduit par une distorsion de la courbe aux très faibles valeurs de $\varepsilon$. Ceci peut se constater sur les figures 8 et 9 où l'on a représenté les barres d'incertitude (dont la valeur est $10^{-5}$ ) sur $\varepsilon$.

2) La dérive propre de la différence de marche dans l'interféromètre, qui introduit une erreur sur la vitesse de la bille. Dans les expériences présentées, nous avons vérifié que cette dérive était au plus de $1 / 4$ de frange pendant la durée de l'acquisition et par conséquent négligeable sur l'intervalle de temps $0,25 \mathrm{~s}$ servant à calculer la vitesse à partir des déplacements. Cette dérive a aussi pour effet de rendre la détermination du point d'arrêt plus délicate.

En fait, la technique expérimentale peut être affinée, en réduisant autant que possible la dérive propre (conception de l'interféromètre et diminution des perturbations thermiques), et en mesurant simultanément le résidu de dérive et le déplacement de la bille. Un gain de l'ordre de un ordre de grandeur en précision/résolution devrait être réalisable.

\section{Conclusion.}

La technique de mesure exposée ici a permis d'obtenir le déplacement de la sphère avec une grande sensibilité (résolution de l'ordre de $5 \times 10^{-9} \mathrm{~m}$ ). On peut obtenir aussi la position 
absolue de l'enveloppe extérieure des rugosités par rapport à la paroi plane, en comptant le nombre de franges ayant défilé de l'instant considéré jusqu'à l'instant où la sphère-touche la paroi.

L'accord avec la théorie de la lubrification est très bon jusqu'à des distances de l'ordre de 10 fois la rugosité (qui est ici environ $1,5 \times 10^{-6} \mathrm{~m}$ ) ou supérieures. L'effet de la rugosité sur le coefficient de frottement est mis en évidence lorsque la distance à la paroi est de l'ordre de grandeur de cette rugosité.

Lorsque cette distance décroît, le comportement non-newtonien du fluide peut aussi avoir une influence. Cette influence est faible ici, mais elle pourrait être étudiée systématiquement avec des liquides présentant un comportement non-newtonien notable.

La technique a permis d'observer aussi le mouvement de la sphère à des distances de la paroi de l'ordre de $10^{-8} \mathrm{~m}$. Nous pensons que, convenablement affinée, la technique interférométrique permet probablement de gagner près d'un ordre de grandeur en précision/résolution et proximité de la paroi. Il serait alors sans doute possible, en utilisant cette technique expérimentale avec des sphères plus lisses, d'observer les effets des forces à courte portée du type van der Waals.

\section{Bibliographie}

[1] AdAmCZyK Z., AdAMCZYK M. and VAN DE VEN T. G. M., Resistance coefficient of a solid sphere approaching plane and curved boundaries, J. Colloid Interface Sci. 96 (1983) 204-213.

[2] Ambari A., Gauthier-Manuel B. and GuYon E., Wall effects on a sphere translating at constant velocity, J. Fluid Mech. 149 (1984) 235-253.

[3] Cox R. G. and BrenNer H., The slow motion of a sphere through a viscous fluid towards a plane surface. II Small gap widths including inertial effects, Chem. Eng. Sci. 22 (1967) 1753-1777.

[4] JOYEUX D., Real time measurement of very small transverse displacements of diffuse objects by random moiré. 1. theory, Appl. Opt. 15 (1976) 1241-1247.

[5] Smart J. R. and Leighton D. T. Jr., Measurement of the hydrodynamic surface roughness of noncolloidal spheres, Phys. Fluids A 1 (1988) 52-60.

[6] Stokes G. G., On the effect of the internal friction of fluids on the motion of pendulums, Trans. Cambridge Philos. Soc. 9 (1851) 8.

[7] YUU S. and FUKUI Y., Measurement of fluid resistance correction factor for a sphere moving through a viscous fluid toward a plane surface, A. I. Ch. E. J. 27 (1981) 168-170. 